\title{
Iteration less Wavelet-Fractal Image Compression Applicable in Cellular Mobile Communication System
}

\author{
Sheeba K. \\ Assoc.professor,LBSCE \\ \& Ph.D Scholar, \\ Karpagam University \\ Coimbatore, India
}

\author{
Abdul Rahiman M. \\ Pro. Vice Chancellor \\ APJ Abdul Kalam Technological \\ University Kerala, India
}

\begin{abstract}
Fractal image compression is a an active area of research with new promising technique that will work very effectively in areas where we have to deal with a huge size of data .In Fractal compression major challenge is the exhaustive comparison needed in the encoding stage. In this paper a method of iteration free- detail space fractal image compression is proposed, in which time of encoding is reduced without compromising much on the quality of image and this algorithm guarantees a high compression ratio. This is a hybrid algorithm of fractal mathematics and wavelet Transform. When comparing with the existing hybrid techniques the advantage of this proposed method is, only the approximation space undergoes exhaustive comparison and thereby it guarantees higher speed than the existing Hybrid techniques. IFS (Iterated function system) of detail space are calculated using the result of approximation space. Experimental results show that in the proposed method Computational over head is considerably reduced and maintains a good tradeoff between compression ratio and quality of image. Resultant image is resolution independent, which is the one of the properties of fractal image compression. Since this new technique guarantees, high compression ratio and low encoding time, it may work very well in mobile communication, especially in face book application.
\end{abstract}

\section{General Terms}

Fractal image compression, Hybrid compression, DWT, BFIC

\section{Keywords}

Affine transformation, fixed attractor, Fractal, Wavelet transform, Iterated function system

\section{INTRODUCTION}

An image is the two dimensional representation of a three dimensional scene. Digital image is the numerical representation of an image. Digital image processing is the process of acquiring and manipulation of an image using a digital processor or computer. Digital image compression is an important area in the digital image processing. In the present world of multimedia application, where we have to deal with a chunk of data, compression has a key role. Attaining a best image quality at a reduced bit rate is the main goal of image compression. Image compression is the technique of representing an image in a compact form without losing the information. We have lot of compression algorithms, but none of them show a good performance where high compression is required. Also none of the existing compression algorithms support zooming of the reconstructed image without compromising the quality of the image, there is a tradeoff between bit rate and quality of image. Transform coding technique like DCT, DWT provides a good tradeoff between these parameters. But applications where we need very high compression ratio ,these techniques are not a good choice. There comes the importantance of fractal image compression. This concept is introduced by M.Barnsley in 1988[1].Fractal theory is entirely different from other algorithms such as DCT,DWT, VQ etc. [2],[3],[4]. In image compression techniques, we exploit the feature of redundancy in the images. Fractals have self similar properties, with a fragmented geometric shape and fractional dimension that can be split into parts. Fractal mathematics is based on "collage theorem" which is proved by Michel Barsley, a Professor of Georgia Tech. [5]

Mathematical approach of fractal method based on block forming was first suggested by Jacquin in 1990[6]. Fractal Image Compression is based on the theory of Iterated Function System [7], [8] which is a collection of contractive affine transformations. Fractal image compression is effective in the case of image which shows high degree of self similarity. So this is an excellent tool to highly compress natural images, which shows high degree of self similarity. Fractal coding is not accepted as an international standard as JPEG and JPEG2000 [9] because of its unsymmetrical property of encoding and decoding phase. Coding process requires rather long time for domain code book generation and domain - range matching process. Comparing with the available present Hybrid Fractal -Wavelet compression [10],[11],this proposed ILFIC compression algorithm provides high compression ratio with symmetric encodingdecoding algorithms without compromising much on the quality of picture.

Wavelet Transform Based Fast Fractal Image Compression which is proposed by R.E Chaudhari [13] is a hybrid approach of joining the multi resolution property of discrete wavelet transform and self similarity property of fractal image compression. Iterated Function system of the high entropy approximation band and low entropy detail coefficient sub bands are constructed separately which improves the encoding time without much degradation in the decoded image quality. This paper combines fractal image coding and multi resolution wavelet transform and proposes a technique of iteration free algorithms in the high frequency sub bands to make the encoding process faster than other algorithms. In the proposed technique compression ratio is kept at a value of 52.5 , and it is shown that the encoding time is improved by a percentage $91.24 \& 24.94$ than that of BFIC and WFC respectively .It is very hopeful that along with the improvement in encoding time, the compression ratio of the proposed method is improved by two times. These result analysis guarantees that encoding- decoding process become almost symmetric in the proposed method. Since this new technique provides a very high compression ratio, with a better PSNR, it will be applicable in sharing images in Face book application which is used in android mobile phone. 


\section{BASIC FRACTAL IMAGE COMPRESSION}

\subsection{What is Fractal Image?}

An image which shows self similarity at different level of scales is named as fractal image. We can see that all natural images exhibit self similarity at different level of scales. Image of fire and image of celestial bodies shown in figure 1 are examples of fractal images. The characteristics of these types of fractal images are that it can be generated by an iterative function system (IFS) starting from any arbitrary image. Fractal images have fractional dimension, it has a simple, possibly recursive definition. This self similarity property contributes the important ability to zoom fractal compressed image without the distortion of pixelization effect. That is fractal image compression is resolution independent and this is the one of the main advantages of fractal compression over standard image compression technique of JPEG.

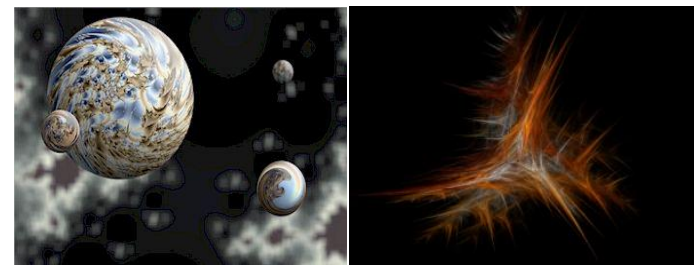

Figure1: Natural images

\subsection{Statement of Collage Theorem}

Fractal image compression is based on the mathematical principle of Collage Theorem. It states that,

Let $(X, d)$ be a complete metric space and take $L \in \mathrm{H}(X)$. Consider an iterated function system; IFS $\left\{X ; \mathrm{w}_{0}\right.$, w1, $\left.\mathrm{w} 2 \ldots \mathrm{w}_{\mathrm{N}}\right\}$ with a contractivity factor $0 \leq \mathrm{s}<1$. Such that

$\mathrm{h}\left(\mathrm{L}, \bigcup_{n=0}^{N} w n(L)\right) \leq \epsilon$, Where $\mathrm{h}(\mathrm{d})$ is the Hausdorff metric. Where $\epsilon>0$

Then $h(L, A) \leq \frac{\epsilon}{1-s}$ Here $A$ is called the attractor of IFS.

$\mathrm{h}(\mathrm{L}, \mathrm{A}) \leq(1-\mathrm{c})^{-1} \mathrm{~h}\left(\mathrm{~L}, \mathrm{U}_{n=0}^{N} w n(L)\right)$ for all $\mathrm{L} \in \mathrm{H}(\mathrm{X})$

\subsection{Contractive Transformation}

A Transformation ' $\mathrm{w}$ ' is said to be contractive if for two points $\mathrm{P}_{1}, \mathrm{P}_{2}$ and the distance

$$
\mathrm{d}\left(\mathrm{w}\left(\mathrm{P}_{1}\right), \mathrm{w}\left(\mathrm{P}_{2}\right)\right)<\mathrm{s} \mathrm{d}\left(\mathrm{P}_{1}, \mathrm{P}_{2}\right) \quad \text { for some } \mathrm{s}<1 \text {, }
$$

Where $d=$ distance. This formula says the application of a contractive map always brings points closer and after some iteration it reaches to a unique fixed point called attractor.In fractal image compression, contractive affine transformation (IFS) is repeatedly applied on an arbitrary image and after some iteration it reaches at a fixed image which is called attractor. Attractor resembles the input image.

\subsection{Matrix form representation of Affine Transformation}

$$
w_{i}\left[\begin{array}{c}
x \\
y \\
z
\end{array}\right]=\left[\begin{array}{ccc}
a_{i} & b_{i} & 0 \\
c_{i} & d_{i} & 0 \\
0 & 0 & s_{i}
\end{array}\right]\left[\begin{array}{l}
x \\
y \\
z
\end{array}\right]+\left[\begin{array}{c}
e_{i} \\
f_{i} \\
o_{i}
\end{array}\right]
$$

The coefficients of affine transformation are adjusted in order to shrink, translate, rotate, and shear the new image, so that it lies over a part of input image. Parameters a,b,c and $d$ represent the linear transformation operation on the domain block to get the best approximation of the chosen range block and $\mathrm{e}$ and $\mathrm{f}$ are the coordinates of the domain block, which shows best correlation with the chosen range block. Here $z$ denote the intensity of the pixel in the location $\mathrm{x}$ and $\mathrm{y}, \mathrm{s}$ and $\mathrm{o}$ are the contrast and brightness adjustments to get the best matching.

\subsection{Eight Affine Transformations in the Dihedral Group}

To each range block, we must calculate the similarity measure with all eight transformed blocks of decimated domain block according to the Dihedral transformations in Table I.

\subsection{JPEG Vs FIC}

JPEG is the most widely used standard image compression technique, so it is very genuine to compare the very competing method of FIC with JPEG. JPEG uses Discrete Cosine Transform coding, which convert highly correlated large set of time domain intensity values in to small set of uncorrelated frequency coefficients. In this technique compression is achieved by discarding high frequency coefficient having low energy and taking lower frequency band where most of the energy is condensed. By varying this truncation frequency, we can make a tradeoff between compression ratio and quality of image .Drawback of this method is that it causes ringing artifacts at the edges and while zooming some blocking artifacts are formed.ie it is a resolution dependent method. FIC is a resolution independent method, and it supports the zooming of the image.

\begin{tabular}{|c|c|c|c|}
\hline Symmetry & Angles & Equations & Results \\
\hline \multirow[t]{2}{*}{ T0 } & \multirow[t]{2}{*}{ Identity } & $\mathrm{x}^{\prime}=\mathrm{x} \cos (0)+\mathrm{y} \sin (0)$ & $\mathrm{x}^{\prime}=\mathrm{x}$ \\
\hline & & $y^{\prime}=-x \sin (0)+y \cos (0)$ & $\mathrm{y}^{\prime}=\mathrm{y}$ \\
\hline \multirow[t]{2}{*}{$\mathrm{T} 1$} & \multirow[t]{2}{*}{$\operatorname{Rot}(+90)$} & $\begin{array}{c}\mathrm{x}^{\prime}=\mathrm{x} \cos (90)+ \\
\mathrm{y} \sin (90)\end{array}$ & $x^{\prime}=y$ \\
\hline & & $\begin{array}{c}\mathrm{y}^{\prime}=- \\
\mathrm{x} \cos (90)+\mathrm{y} \sin (90)\end{array}$ & $y^{\prime}=-x$ \\
\hline \multirow[t]{2}{*}{$\mathrm{T} 2$} & \multirow[t]{2}{*}{$\operatorname{Rot}(+180)$} & $\begin{array}{c}\mathrm{x}^{\prime}=\mathrm{x} \cos (180)+ \\
\mathrm{y} \sin (180)\end{array}$ & $\mathrm{x}^{\prime}=-\mathrm{X}$ \\
\hline & & $\begin{array}{c}y^{\prime}=-x \sin (180)+ \\
y \cos (180)\end{array}$ & $y^{\prime}=-y$ \\
\hline \multirow[t]{2}{*}{ T3 } & \multirow[t]{2}{*}{$\operatorname{Rot}(270)$} & $\begin{array}{c}\mathrm{x}=\mathrm{x} \cos (270)+ \\
\mathrm{y} \sin (270)\end{array}$ & $\mathrm{x}^{\prime}=-\mathrm{y}$ \\
\hline & & $\begin{array}{c}\mathrm{y}^{\prime}=\mathrm{x} \sin (270)+ \\
\mathrm{y} \cos (270)\end{array}$ & $\mathrm{y}^{\prime}=\mathrm{x}$ \\
\hline \multirow[t]{2}{*}{$\mathrm{T} 4$} & \multirow[t]{2}{*}{$\begin{array}{l}\text { Ref. at } \mathrm{x}^{-} \\
\text {axis }\end{array}$} & $x^{\prime}=-x \cos (0)+y \sin (0)$ & $\mathrm{x}^{\prime}=-\mathrm{x}$ \\
\hline & & $y^{\prime}=-x \sin (0)+y \cos (0)$ & $y^{\prime}=y$ \\
\hline
\end{tabular}

Table 1: Linear Transformations on the domain block 


\begin{tabular}{|c|c|c|c|}
\hline \multirow[t]{2}{*}{ T5 } & \multirow[t]{2}{*}{$\begin{array}{c}\text { Ref } \\
\& \operatorname{Rot}(-90)\end{array}$} & $\begin{array}{c}\mathrm{x}^{\prime}=\mathrm{x} \cos (90)+ \\
\mathrm{y} \sin (90)\end{array}$ & $x^{\prime}=-y$ \\
\hline & & $\begin{array}{c}y^{\prime}=-x \sin (90)+ \\
y \cos (90)\end{array}$ & $y^{\prime}=-x$ \\
\hline \multirow[t]{2}{*}{ T6 } & \multirow[t]{2}{*}{$\begin{array}{c}\text { Ref. \& } \\
\operatorname{Rot}(180)\end{array}$} & $\begin{array}{c}\mathrm{x}^{\prime}=-\mathrm{x} \cos (180)+ \\
\mathrm{y} \sin (180)\end{array}$ & $\mathrm{x}^{\prime}=\mathrm{x}$ \\
\hline & & $\begin{array}{c}y^{\prime}=x \sin (180)+ \\
y \cos (180)\end{array}$ & $y^{\prime}=-y$ \\
\hline \multirow[t]{2}{*}{$\mathrm{T} 7$} & \multirow[t]{2}{*}{$\begin{array}{c}\text { Ref \& } \\
\operatorname{Rot}(270)\end{array}$} & $\begin{array}{c}\mathrm{x}^{\prime}=-\mathrm{x} \cos (270)+ \\
\mathrm{y} \sin (270)\end{array}$ & $\mathrm{x}^{\prime}=\mathrm{y}$ \\
\hline & & $\begin{array}{c}\mathrm{x}^{\prime}=-\mathrm{x} \cos (270)+ \\
\mathrm{y} \sin (270)\end{array}$ & $y^{\prime}=x$ \\
\hline
\end{tabular}

But the main drawback of FIC comparing with the standard compression algorithm is that the unsymmetrical encoding and decoding phase.

\section{BLOCK DIAGRAM OF THE PROPOSED METHOD (ILFIC)}

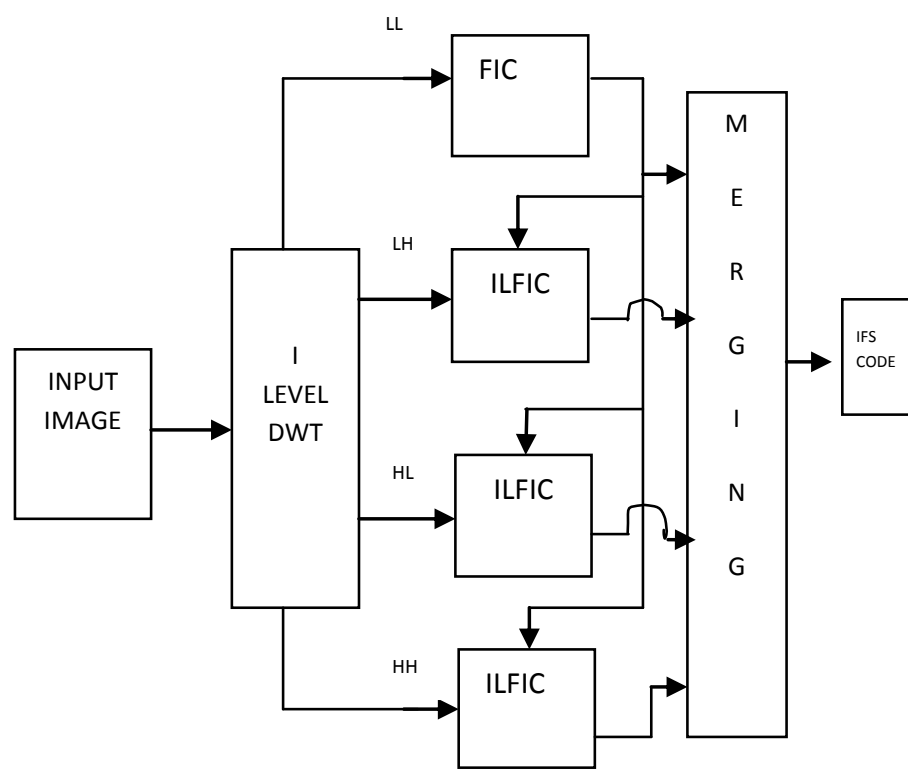

Figure 2: Block Diagram

\section{ALGORITHM OF PROPOSED METHOD (ILFIC)}

\subsection{Encoding Phase}

1. Read the image

2. Perform two dimensional DWT on the image

3. Divide the four sub bands into non overlapping range block $(\mathrm{R})$ of sizeBxB. The parameter $\mathrm{B}$ is commonly taken as two or four

4. Decimate all sub bands band by 2 , Divide it in to overlapping domain block of same size
5. Create 8 linear affine transformations of each domain block, save it in the domain pool(refer table 1)

6. Find the best suiting affine transformation for each range block in the LL band. Save it as IFS1

7. Perform Iteration free Fractal Image Coding (IFFIC) on each high frequency band (LH, HL and $\mathrm{HH}$ ). Here domain coordinates and linear transformation of the LL sub band is referred to get a best match to each range block in high frequency sub band. Save this as IFS2,IFS3 and IFS4

8. Merge IFS (1), IFS (2) IFS (3) and IFS (4) to get the whole fractal code of the original image.

The parameters of transformation such as contrast and brightness are adjusted using the following equation for the best accordance

$$
\begin{gathered}
\mathrm{o}=\frac{\sum \sum(R . D)}{\sum \sum D \cdot D} \\
\mathrm{~s}=\frac{\sum \sum(R)}{B \times B}
\end{gathered}
$$

The distance between a range block $\mathrm{R}$ and a selected decimated domain block $\mathrm{D}$, both with same number of pixel is

$\mathrm{E}(\mathrm{R}, \mathrm{D})=|(\mathrm{S} . \mathrm{D}+\mathrm{O}-\mathrm{R})|$

\subsection{Decoding Phase}

1. Choose an Arbitrary Image

2. Do a one level Discrete Wavelet Decomposition

3. Apply IFS1,IFS2,IFS3 and IFS4 on LL,LH,HL and $\mathrm{HH}$ respectively for a minimum of 10 times

4. Take Inverse Discrete Wavelet Transform

$$
\operatorname{PSNR}=10 * \log \frac{255^{2}}{\frac{1}{N^{2}} \sum_{i=1}^{N} \sum_{j=1}^{N}(f(i, j)-g(i, j))^{2}}
$$

\section{RESULT AND DISCUSSION}

The experiments are simulated using MatLab12 Intel core2duo $1.2 \mathrm{GHz}$ processor. Haar wavelet is used for wavelet decomposition. The results are compared with basic fractal image compression (BFIC) and Hybrid wavelet-fractal image compression (WFC) Experimental results pertaining to six images having size $512 \times 512$ are discussed here. Figure 3 shows the input images and figure 4 shows the resultant images using the proposed method. It can be seen that visual quality of the reconstructed image has not degraded much by applying this new technique at a high compression ratio of 52.5 , so it can be suggested that this is applicable in mobile phone communication system for image transferring. The PSNR, No of MSE calculations and encoding time are computed to determine degree and efficiency achieved in representing the image and amount of reduction in encoding time to analyze the encoding and decoding phase symmetry.

From the table 2 and from the bar charts, it is observed that there is a performance improvement of $91.242 \%$ in encoding time, $21.141 \%$ in no of mse calculations in the proposed method over BFIC and $24.949 \%$ in encoding time, $36.558 \%$ in no mse calculations in the proposed method over the recent technique of WFC. Encoding time is reduced by a value of $58 \%$ on an average and this make sure that in the proposed 
method encoding and decoding process is more symmetric comparing with the previous algorithms.

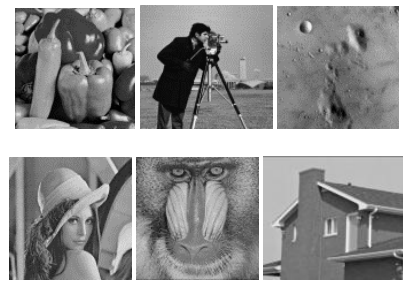

Figure3: Original images
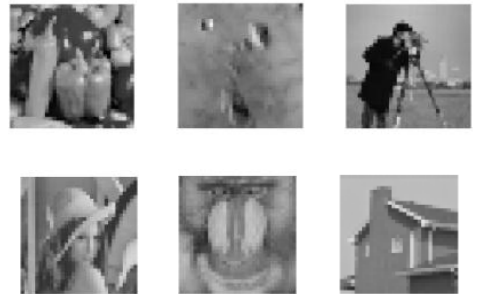

Figure4: Reconstructed image using the proposed method

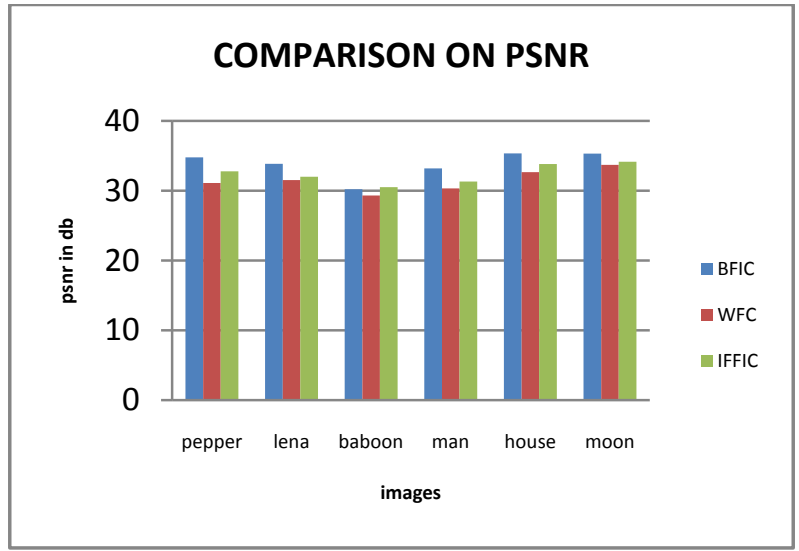

Figure4:Comparison on the parameter, PSNR of proposed method with that of existing techniques

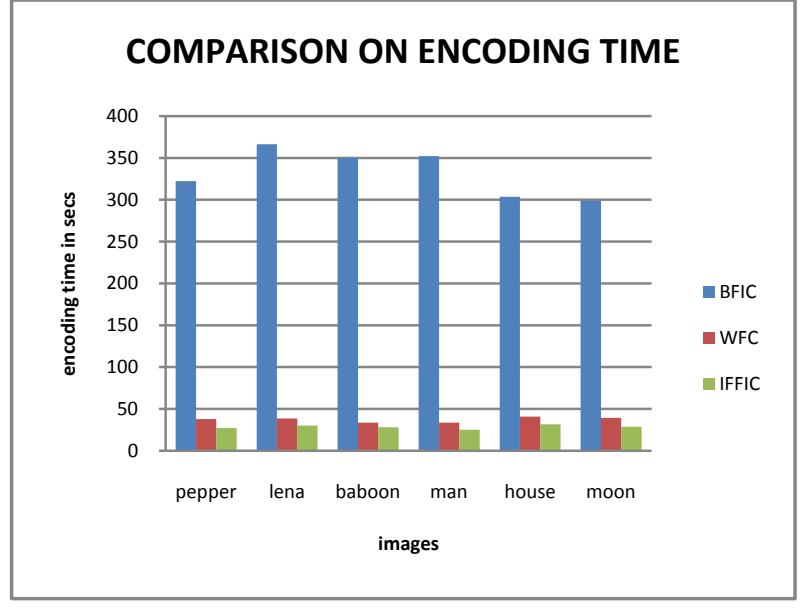

Figure4:Comparison on the parameter, encoding time of proposed method with that of existing techniques

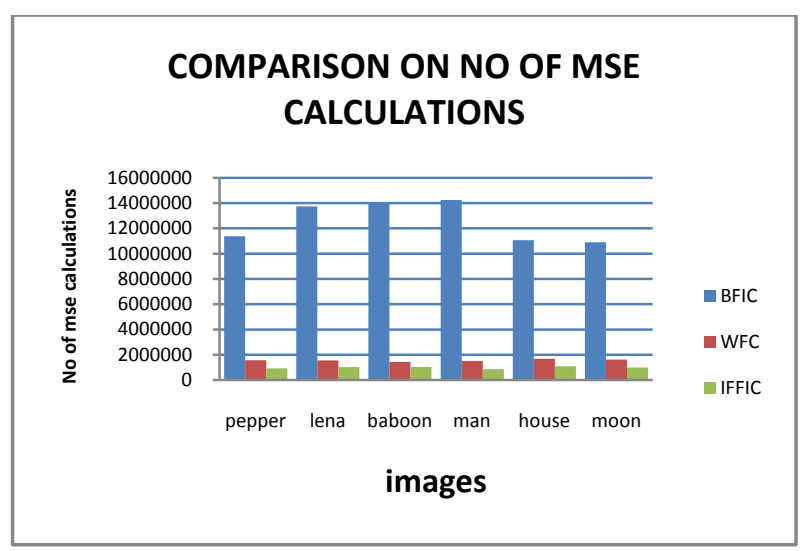

Figure4:Comparison on the parameter MSE calculations of proposed method with that of existing techniques

Table 2. Comparison of existing methods with ILFIC

\begin{tabular}{|c|c|c|c|c|c|c|c|c|c|c|c|c|}
\hline \multirow{2}{*}{$\begin{array}{l}\text { Name } \\
\text { of } \\
\text { image }\end{array}$} & \multicolumn{4}{|c|}{ BFIC } & \multicolumn{4}{|c|}{ WFC } & \multicolumn{4}{|c|}{ ILFIC } \\
\hline & $\begin{array}{l}\text { No of mse } \\
\text { computation }\end{array}$ & PSNR & $\begin{array}{l}\text { Encoding } \\
\text { time }\end{array}$ & $\begin{array}{l}\text { Compr } \\
\text { ession } \\
\text { Ratio }\end{array}$ & $\begin{array}{l}\text { No of mse } \\
\text { computation }\end{array}$ & PSNR & $\begin{array}{c}\text { Encoding } \\
\text { time }\end{array}$ & $\begin{array}{l}\text { Compress } \\
\text { ion Ratio }\end{array}$ & $\begin{array}{l}\text { No of mse } \\
\text { computation }\end{array}$ & PSNR & $\begin{array}{c}\text { Encoding } \\
\text { time }\end{array}$ & $\begin{array}{l}\text { Compress } \\
\text { ion Ratio }\end{array}$ \\
\hline pepper & 11383080 & 34.77 & 322.32 & 25.6 & 1572904 & 31.11 & 37.97 & 25.6 & 925384 & 32.78 & 27.12 & 52.5 \\
\hline lena & 13743680 & 33.86 & 366.27 & 25.6 & 1546056 & 31.51 & 38.61 & 25.6 & 1030808 & 31.99 & 30.15 & 52.5 \\
\hline baboon & 13966216 & 30.20 & 350.29 & 25.6 & 1436528 & 29.31 & 33.59 & 25.6 & 1041800 & 30.50 & 28.21 & 52.5 \\
\hline $\operatorname{man}$ & 14258728 & 33.17 & 352.207 & 25.6 & 1503656 & 30.34 & 37.69 & 25.6 & 864104 & 31.31 & 25.26 & 52.5 \\
\hline house & 11072064 & 35.35 & 303.617 & 25.6 & 1679936 & 32.65 & 40.75 & 25.6 & 1086064 & 33.81 & 31.64 & 52.5 \\
\hline Moon & 10902912 & 35.32 & 298.842 & 25.6 & 1624148 & 33.69 & 39.25 & 25.6 & 992000 & 34.16 & 28.61 & 52.5 \\
\hline
\end{tabular}


Table 3. Performance improvement of the proposed methodover existing algorithms

\begin{tabular}{|c|c|c|}
\hline Parameters & $\begin{array}{c}\text { Performance of } \\
\text { proposed } \\
\text { method(IFFC) } \\
\text { over BFIC }\end{array}$ & $\begin{array}{c}\text { Performance } \\
\text { of propose } \\
\text { method } \\
\text { (IFFIC) over } \\
\text { WFC }\end{array}$ \\
\hline $\begin{array}{c}\text { Encoding } \\
\text { Time }\end{array}$ & $91.242 \%$ & $24.949 \%$ \\
\hline PSNR & $-4.012 \%$ & $2.617 \%$ \\
\hline $\begin{array}{c}\text { No of mse } \\
\text { computation }\end{array}$ & $21.141 \%$ & $36.558 \%$ \\
\hline $\begin{array}{c}\text { Compression } \\
\text { ratio }\end{array}$ & $\begin{array}{c}\text { Improved by a } \\
\text { factor of } 2\end{array}$ & $\begin{array}{c}\text { Improved by a } \\
\text { factor of } 2\end{array}$ \\
\hline
\end{tabular}

\section{CONCLUSION}

Although a lot of papers has been published on the topic of fractal image compression, none of them were accepted as a standard algorithm as JPEG or JPEG2000. The reason is the increased encoding time, even if it provides a good compression ratio among other existing algorithms. This proposed method provides a fairly god tradeoff between compression ratio and quality of image with a reduced encoding time. This Iteration less fractal image compression technique provides a good tradeoff between image quality and compression ratio than the recent technique of Wavelet fractal Image Compression. Also the encoding time is reduced much comparing with the existing Fractal coding techniques

\section{REFERENCES}

[1] M.F. Barnsley, and S. Demko, "Iterated function systems and the global construction of fractals", Proc. Roy. Soc. Lond., vol. A399, pp.243-275, 1985.

[2] F . M. Bayer and R. J. Cintra, Member, IEEE, "Image Compression Via a Fast DCT Approximation", IEEE atin America Transactions , VOL. 8, NO. 6, DECEMBER 2010.

[3] Salam Benchikh, Michael Corinthios, Life ," Efficiency Evaluation of Different Wavelets for Image Compression" The 11th International Conference on Information Sciences, Signal Processing and their Applications: Special Sessions .2012 IEEE.

[4] Hiroki Matsumoto, Student Member, IEEE, Kazuya Sasazaki, and Yukinori Suzuki, Member, IEEE "Color Image Compression with Vector Quantization”, IEEE Conference on Soft Computing in Industrial Applications (SMCia/08), June 25-27, 2008, JAPAN.

[5] M. Barnsley, and A.D. Sloan, "A better way to compress images,"BYTE Magazine, pp. 215-223, 1998.

[6] A. E. Jacquin, "Image coding based on a fractal theory of iterated contractive image transformations" ,IEEE Trans. on Image Processing, Vol. 1, No. 1, pp. 18(30, Jan. 1992.).
[7] Chol-Hui Yun,Metzler W and Barski.M(16-19 June,2008) 'Image Compression Predicated on Recurrent Iterated Function System'.International conference on Mathematics and Statistics.

[8] Fisher Y (1994) . Fractal Image Compression - Theory and Application New York : Springer - Verlag

[9] Nirbhay Kashyap,Dr.Shailendra Narayanan Singh," Review of Image Compression and comparison of it's Algorithms", International Journal of Application or innovation in Engineering \& Management.Vol.2,Issue 12,December 2013.

[10] Andreopoulos,Y.A Karayiannis,T.Stouraitis, "A Hybrid Image Compression Algorithm based on Fractal Image Coding and Wavelet transform",ISCAS 2000-IEEE international symposium on circuits and systems, May 28-31,2000.

[11] Yuzo lano ,Fernando Silvestre da Silva,Ana Lucia Mendas Cruz,"A fast and Efficient Hybrid FractalWavelet Image Coder",IEEE Transactions on Image Processing,Vil.15,No.1,January 2006.

[12] Hitashi,Ganganpreet Kaur,Sugandha Sharma,'Fractal Image Compression a Review", International Journal of Advanced Research in Computer Science and Software Engineering. Vol.2,Issue 2.February 2012.

[13] R.E Chaudhar,'Wavelet Transformed based Fractal Image Compression',International Conference on Circuits,Systems,Communication and Information Technology Application.2014,

[14] Tahamohammed Hassan and XingianWu, (2013).'An Adaptive Fractal Image Compression', International Journal of Computer Science Issue, Vol.10,Issue2,

[15] Yin-Lon Lin and Wen-Lin Chen, (2012) ,Fast Search Strategies for Fractal Image Compression',Journal of Information Science and Engineering,. 